\title{
Ultrasonic Testing Technology of Boiler \&Pressure Vessel
}

\author{
Min $\mathrm{Ji}^{1, \mathrm{a}}$, Jingfeng $\mathrm{He}^{2, \mathrm{~b}}$, Pengfei $\mathrm{Ji}^{3, \mathrm{c}}$ \\ ${ }^{1}$ Xijing University, Shaanxi Xi'an,710123,China \\ ${ }^{2}$ Xijing University, Shaanxi Xi'an,710123,China \\ ${ }^{3}$ China Railway 21 Bureau Group Forth Engineering Co. Ltd., \\ Shaanxi, xi 'an 710065 ,China \\ âE-mail:995010771@qq.com,, E-mail:573825625@qq.com, \\ cE-mail: 597329947@qq.com
}

Keywords: Boiler pressure vessel; Ultrasonic testing

\begin{abstract}
Combined with the analysis of the principle of ultrasonic inspection technology, from the fillet weld of pressure vessel, pressure vessel wall crack and pressure vessel head tube plate crack ultrasonic inspection technology is discussed in the application of the boiler pressure vessel defect detection.

At present, the majority of the boiler pressure vessel in service to take over the fillet weld is in not check status, potential danger. Strengthen the ultrasonic detecting technology to explore to improve the quality of boiler pressure vessel defect detection. Improving the effect of container operation is of great importance.
\end{abstract}

\section{The principle of ultrasonic testing technology}

Ultrasonic testing is mainly used the ultrasonic propagation characteristics in materials, as well as ultrasonic detection and the interaction mechanism of the defects. Theoretically, ultrasound in infinite homogeneous medium is spread along a straight line, but in fact, any medium always have boundaries, when the ultrasonic wave propagation in inhomogeneous medium, or from one medium to another medium, due to the acoustic impedance of the change in medium, ultrasonic will be produced on the surface of the acoustic impedance change of boundary reflection, refraction and transmission phenomenon, so as to judge whether the detected object.

At present, the most widely used in ultrasonic detection is A-pulse detector. Ultrasonic testing practice through long-term and full understanding of materials, manufacturing process, through the study of the anatomy of defects found in testing, analysis, validation, to accumulate experience. It can from the display screen that shows static and dynamic of defect echo waveform, the wave speed, the echo of steep and echo along the down slope, wave shape, the echo of wide, defect echo amplitude while moving the probe, location, quantity and shape of the envelope, the bottom of the multiple reflection wave frequency and amplitude of the decline law of bottom wave height loss and so on, and the size of the defect equivalent, the extension situation, combining with the characteristics of concrete materials, technology and ultrasonic basic theoretical knowledge to make comprehensive judgment, to accurately evaluate defects nature or kind. In the boiler pressure vessel 
inspection, ultrasonic are the obvious advantages, namely: (1) Ultrasonic directivity is good, it can form a narrow beam. (2) A wavelength, small defects can also be a good reflection. (3) The distance resolution and the resolution of the defects are well. Because of crack is one of the most dangerous defects by ultrasonic testing can greatly improve the defect detection rate, and to ensure product quality.

\section{The common defects of boiler and pressure vessel}

Crack. Crack is a kind of dangerous defects. as long as pressure vessel standards, the judge of crack is not qualified. Crack formation mechanism is complex, according to the conditions and time, it can be divided into: hot crack and reheat crack, cold crack and lamellar tearing. Crack direction is very strong, some echo is bigger, but some are small echo, with different $\mathrm{K}$ value probe, from a different direction incidence, we may find bigger echo.

Not fusion. Incomplete fusion refers to the weld metal and base metal or weld metal that did not melt together. Not fusion weld according to their parts can be divided into groove, incomplete fusion and root incomplete fusion between the layers.

Incomplete fusion on the one hand, can according to their location decision; On the other hand, because of its for planar defects, directionality is very strong. Therefore, different $\mathrm{K}$ value probe, the echo of the equivalent can have very big difference.

Lack of penetration. Lack of penetration is lack of penetration between lack of penetration and the root, especially for the root of lack of penetration, the echo wave speed, strong reaction, on both sides of weld detection can be found, and the reflection amplitude are roughly the same.

\section{Boiler pressure vessel of ultrasonic testing technology}

The boiler pressure vessel fillet weld ultrasonic testing technology. Diagonal weld for the ultrasonic testing of all product generally uses twin straight probe and angle probe to test methods. Twin straight probe test, detection twin probes in to take over the wall, easy to check out at this time near the probe on the surface of the side and shell side groove incomplete fusion. For lack of penetration weld root class discontinuity can't check out and twin straight probe not testing are done by angle probe and inspection.

Probe selection:

Diagonal weld ultrasonic testing, we usually take over side using the direct method and a reflection method, in order to ensure that after small tube wall reflection of the ultrasonic beam that still has high detection sensitivity and high resolution. Suggestions according to the different thickness of collection box and the takeover, we adopt the focus of specially developed different focal length of path line Angle probe or double crystal focusing Angle probe small tube.

The determination of inspection surface:

Due to the effect of structure, diagonal shear wave testing of welds, only option on the each side of the inside and outside the tube plate do the single side inspection. In front of the shear wave detection, with a span formula of reflection method draw line probe from the weld area.

High more than can be found for fillet weld and heat affected zone of possible defects, then this value add fillet weld high waist and heat affected zone. According to the principle of triangulation, we can draw a the inspection probe moving distance of the line, to ensure that in the double span beam can complete check to fillet weld area. Lack of penetration testing root can be defined by a 
direct method of baseline that is $22.5 \mathrm{~mm}$. Twin straight probe were found in mobile area in the boiler header circumferential curvature model in the header of the endplate and tube plate to create fillet weld width, and the heat affected zone of $5 \mathrm{~mm}$.

Scan lines and sensitivity calibration adjustment:

Based on surface flat bottom hole block adjustment instrument line, because the wall thickness is more drop $100 \mathrm{~mm}$, so scan baseline adjusted according to the depth of 1:2.Downcomer fillet weld testing thickness is the largest thickness, angle of weld width and weld on both sides of the covering heat affected zone width (generally take $5 \mathrm{~mm}$ ) combined.

The boiler pressure vessel wall crack ultrasonic testing. Boiler pressure vessel wall crack principle of ultrasonic detection is based on the existing conditions of test equipment, combined with the specifications of the work piece tested, the structure shape and crack inevitably form, proposed using small $\mathrm{K}$ value and the $\mathrm{K}$ value, the combination of two kinds of angle probe ultrasonic testing plan, to determine whether the container wall stress cracking.

To the same container inspection successively uses $\mathrm{K} 1$ and $\mathrm{K} 3$ probe to scan, and mutual authentication for defects. With K1 probe was carried out on the tube along the longitudinal and circumferential scanning, circumferential scanning probe is difficult to achieve a good coupling, so given priority to with axial scan. Such as the wall there is a turtle shape crack, K1 probe scan shows for the depth of the wall thickness of end angle of reflection wave, the wave height with the thickness of block corner reflection close. Under the scanning sensitivity for the crack depth roots often without reflection.K1 probe scanning purpose is to determine whether the lining mouth, crack depth quantitative difficulties. Screen shows the takanami is always in the depth of the wall thickness of position, and as the movement of the probe, defect wave appear constantly. With K3 probe to scan header, circumferential and longitudinal, give priority to with longitudinal scan. When week scan up because of the influence of the shape structure, qualitative and positioning analysis of the reflected wave is difficult.

In the root systems of crack propagation, reflection wave is closer to the vertical reflection and signal is the strongest. Display shows the reflection wave of cracking is the main root. In the case of crack distribution is more dense, before and after the probe moves, due to the crack defects adjacent wave interference, pipe inner wall of the reflection wave disappeared, and the other of the reflection crack root into the screen, crack depth on the crack for quantitative.

Defects of quantitative measurement and evaluation:

Found defect wave detection, the method of using, moving the probe before and after, to find the highest echo of defects. Then according to the different probe set by defect quantity, straight probe and to the flat bottom hole set flaw echoes in equivalent value, $\mathrm{K} 1$ and $\mathrm{K} 3$ angle probe detection, according to the detection sensitivity set high defect echo in the distance a band area and high decibel value.

\section{Boiler pressure vessel ultrasonic detection technology in the future}

As the temperature changes, ultrasonic sound pressure attenuation change is significant. In addition, different reflector may have different attenuation amplitude, and frequency of different sensors have different attenuation characteristics. These questions are needed to strengthen in the process of high temperature ultrasonic testing in the future research and attention. In addition, when the probe and the sensitivity test block and ground work piece surface coupling condition is not at the same time, 
without proper compensation, also it can make quantitative error increases and the accuracy of decline.

At present, some of the structure of the ultra-high pressure vessel elements in the process of using could not detect, it need to continue to develop new technologies and methods of nondestructive testing (NDT) methods. Because of the shape, it need to carry a variety of detection equipment developed testing large length to diameter ratio of the inner wall of the container of creeping device.

\section{References}

[1]ErvinSejdi‘e1.D.e.JinJiang.Timeequencyfeatuxere Presentationusingenergyconcentration: AnoVer viewofrecentadvanees [J].DigitalSignalProeessing.200919: 153 18.

[2]AbidAliShah,Y.R .Non-destruetivemeasurements of erae kasses smeniand defeedeteetionineonere testructures[J].MaterialsandDesign.2008, 29:61-69.

[3]Hui-xing Yun. Damage detection based-propagation of the longitudinal guided wave in a bimetal composite pipe $[\mathrm{J}]$. Mechanic Automation and Control Engineering (MACE). Hohhot,2011:134-138. 